Type of the Paper (Original Article)

\title{
Responsive signatures established by pharmaco- transcriptomic correlation analysis identifies subsets for PARP-targeted therapy and reveals potential synergistic interactors
}

\author{
Haitang Yang1,*, Beibei Sun ${ }^{2}$, Sean R R Hall ${ }^{3}$, Ke Xu' ${ }^{1}$, Liang Zhao ${ }^{4}$, Swee T. Tan ${ }^{3}$, Ralph A. Schmid ${ }^{4}$, \\ Ren-Wang Peng ${ }^{4}$, Feng Yao ${ }^{1, *}$ \\ 1 Department of Thoracic Surgery, Shanghai Chest Hospital, Shanghai Jiao Tong University, Shanghai, \\ People's Republic of China. \\ 2 Institute for Thoracic Oncology, Shanghai Chest Hospital, Shanghai Jiao Tong University, Shanghai, People's \\ Republic of China. \\ ${ }^{3}$ Gillies McIndoe Research Institute, Wellington, New Zealand. \\ ${ }^{4}$ Division of General Thoracic Surgery, Department of BioMedical Research (DBMR), Inselspital, Bern \\ University Hospital, University of Bern, Switzerland. \\ * Correspondence: feng.yao@shchest.org OR yaofeng6796678@126.com (F.Y.); haitang.yang@dbmr.unibe.ch OR \\ xyxm8889@163.com (H.Y.).
}

Received: date; Accepted: date; Published: date

\begin{abstract}
Poly (ADP-ribose) polymerases (PARPs) have pleiotropic roles including canonical DNAdamage response pathways in tumors. Targeting PARP has gained increased attention to treat various cancer types. However, biomarkers predicting sensitivity/resistance to PARP inhibitors (PARPis) and cancer subtypes vulnerable to PARP targeted therapies remain to be defined. Through implementing integrative pharmaco-transcriptomic analyses by correlating the drug response profiles of clinicallyapproved PARPi Olaparib with the transcriptomes of solid cancer cell lines ( $n=659)$, we establish PARPis responsive gene signatures with high reproductivity. With the signatures, we identify tumor subsets vulnerable for PARPi and several potential targets synergistically interacting with PARPi.
\end{abstract}

Keywords: PARP; gene signature; cancer subtype; targeted therapy; synergism

\section{Introduction}

Genomic instability is a hallmark of cancer [1]. Poly(adenosine diphosphate-ribose) polymerase (PARP) is a critical component of the single-stranded break (SSB) repair pathway. PARP comes into focus as a target when SSB repair was identified as a synthetic lethal partner with homologous recombination deficiency (HRD) that is typically induced by BRCA1/2 mutations [2]. Beyond SSB repair, increasing evidence has also suggested that PARP is implicated in numerous other biological processes, such as oxidative stress response and mitochondrial homeostasis [3,4]. As such, recently there has been increasing evidence supporting the expansion of PARP-targeted therapy beyond HRD [5-7]. However, biomarkers predicting sensitivity/resistance to PARP inhibitors (PARPis) and cancer subsets vulnerable to PARP targeted therapies remain to be defined [8]. Additionally, synthetic lethality pathways for PARP-targeted therapy are being studied extensively due to the rapidly developed resistance to PARP inhibitors (PARPi) $[9,10]$, which requires further investigations.

In this study, we sought to systematically delineate the gene networks correlated with PARPi sensitivity and resistance in solid tumor cells to identify the responsive biomarkers to PARP targeted therapies. Based on comprehensive correlation analysis of drug response profiles with genome-wide transcriptomics across solid cancer cell lines $(\mathrm{n}=659)$, we established PARPis sensitive and resistant 
signatures, which then leads to identifying cancer subtypes that are suitable for PARPis-based therapy. More importantly, the integration of the PARPis resistant signature informed several molecular inhibitors that potentially synergize with PARPis.

\section{Results}

2.1. Systematic correlation analysis identifies gene signatures predicting the sensitivity and resistance to clinically-approved PARPi Olaparib

To systematically identify gene signatures that predict Olaparib response, we correlated the sensitivity profiling of Olaparib against the transcriptomes of pan-solid cancer cell lines $(n=659)$, based on a previously curated small-molecule compound library screen dataset (Figure 1A) (17). The results revealed two gene signatures that were significantly correlated (empirical $\mathrm{p}$-value $<0.05$ ), negatively (Figure 1B) or positively (Figure 1C), with the area under the curve (AUC), a measure of drug sensitivity determined by fitted concentration-response curves, of Olaparib. Of note, the negatively correlated genes indicate that higher gene expression correlates with lower AUC value and thus are sensitive to Olaparib. In contrast, the positively correlated genes indicate that higher gene expression correlates with a higher AUC value and thereby is resistant to Olaparib. Thus, we defined the negatively correlated genes as PARPi sensitive signature, containing 30 genes, and the positively correlated genes as PARPi resistant signature, containing 30 genes (Figure 1B, C). 
A

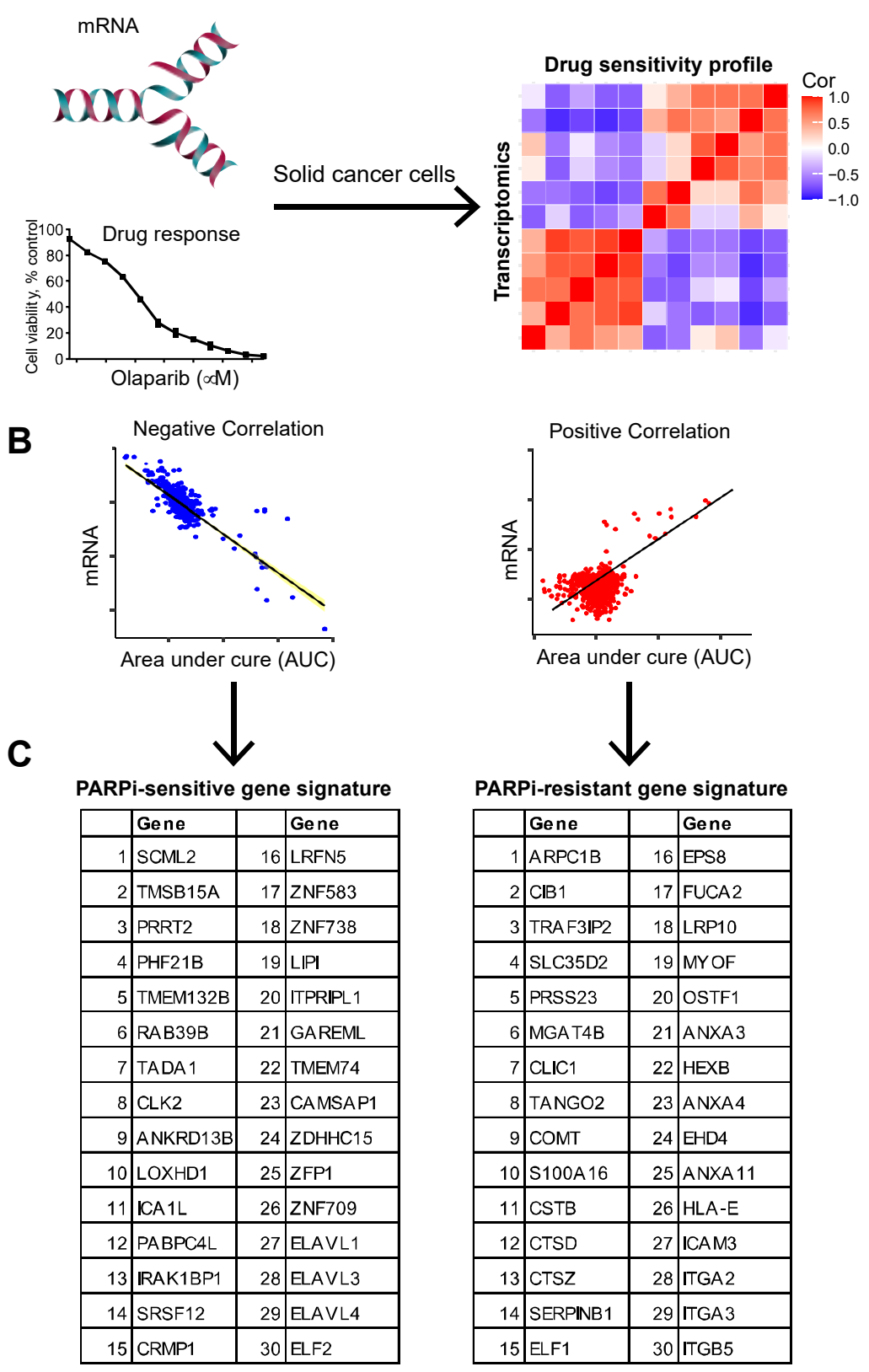

Figure 1. Systematic correlation identifies PARPi sensitive and resistant gene signatures. A, Schematic model shows the pharmacogenomic analysis that integrates transcriptomic data and drug response profiles of clinicallyapproved PARP inhibitor (PARPi) Olaparib across pan-solid cancer cell lines ( $n=659)$; B-C, Genes in PARPi sensitive (in blue, left in B-C) and resistant (in red, right in B-C) signatures were established.

\subsection{Reproductivity of the established PARPi sensitivity and resistance gene signatures}

To confirm the reproductivity of our established gene signatures, we applied them to an independent cohort GDSC (Genomics of Drug Sensitivity in Cancer, https://www.cancerrxgene.org/), which provides sensitivity data of hundreds of drug compounds, including several clinically-approved PARPis, e.g. Olaparib, Talazoparib, and Niraparib, assayed on hundreds of cancer cell lines from CCLE (Cancer Cell Line Encyclopedia) cohort. We first calculated PARPi sensitive and resistance gene signature scores across pan-solid cancer cell lines, which were then merged with the drug sensitivity profiles from GDSC. The results showed that the AUC values of the PARPis Olaparib, Talazoparib, and 
Niraparib were all significantly negatively correlated with the PARPi gene signature score (Figure 2A), and positively correlated with the PARPi resistant gene signature score (Figure 2B), confirming the reproductivity of our established PARPi signatures. Additionally, we explored the association of the PARPi signatures with a well-curated HRD signature score [11], which, intriguingly, demonstrated that there are heterogeneous correlation patterns between the PARPi signatures and the HRD signature score across the TCGA pan-cancer patient cohort (Figure 2C, D). The results suggest that the PARPi signatures might be independent of the HRD signature. In line with this, recent studies support the expansion of PARP-targeted therapy in cancer irrespective of classical HRD signatures [5-7].

A
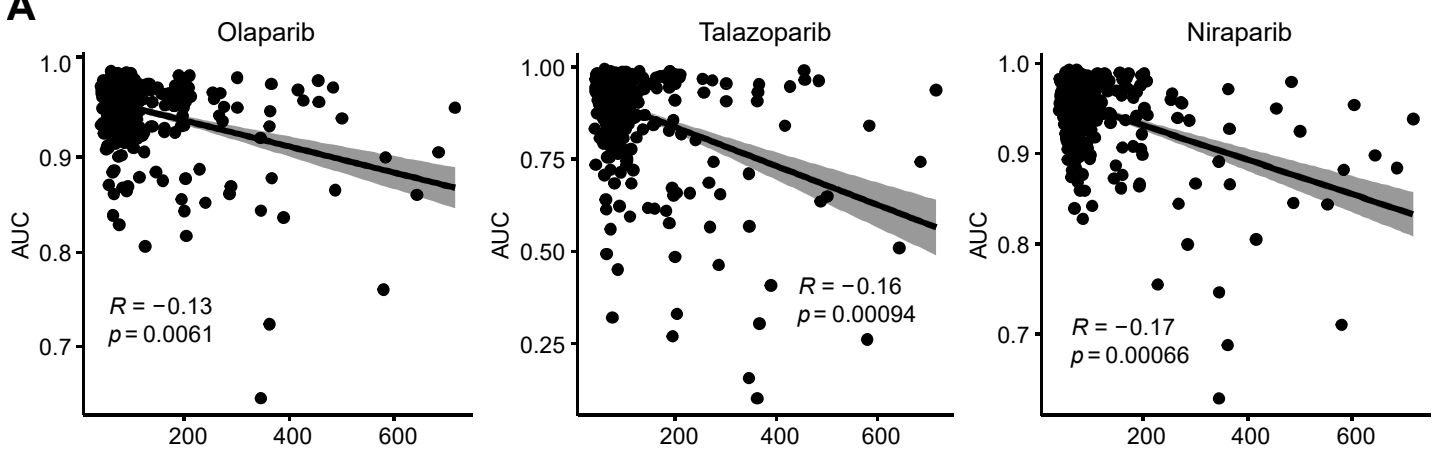

B

PARPi sensitive signature score
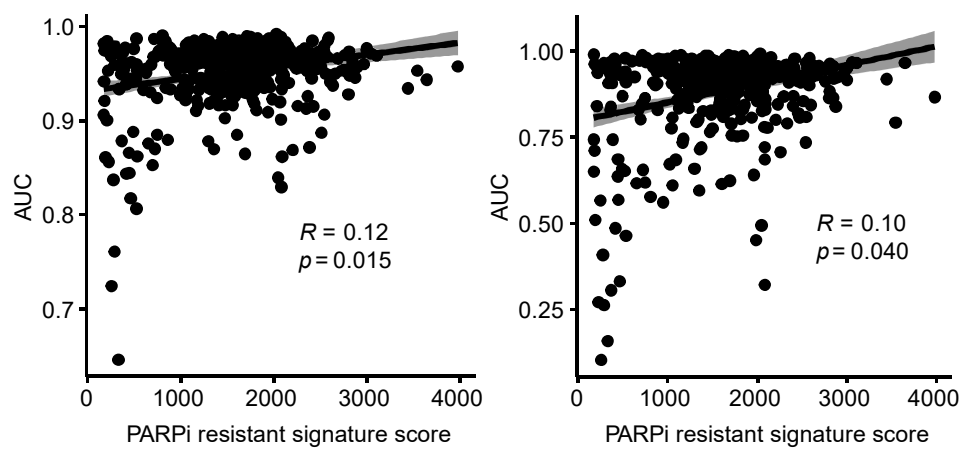

PARPi sensitive signature score
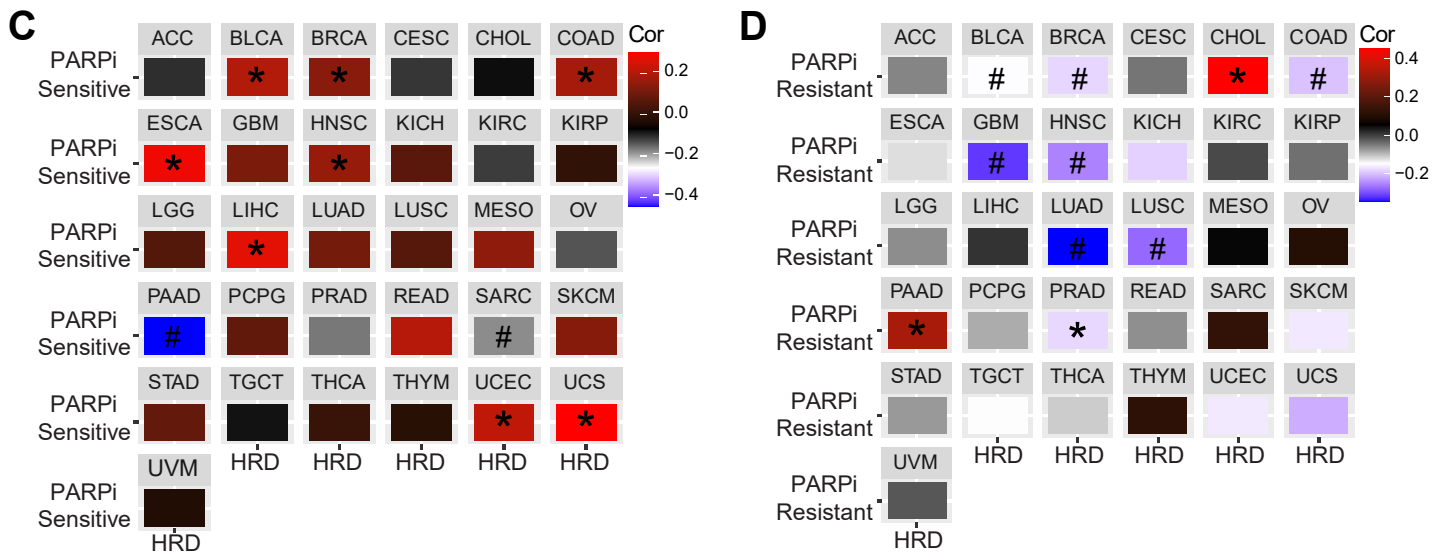

Figure 2. Reproductivity of the PARPi gene signatures. A-B, An independent data cohort validating the correlation of PARPi sensitive (A) and resistant (B) signatures with the AUC (area under the curve) values of three clinically-approved PARPis (Olaparib, Talazoparib, and Niraparib). Drug response profiles of the three PARPis were downloaded from the GDSC (Genomics of Drug Sensitivity in Cancer; https://www.cancerrxgene.org/) database, and were integrated with the PARPi sensitive (A) and resistant (B) signature scores across CCLE (Cancer Cell Line Encyclopedia) pan-solid cancer lines; C, Association of PARPi sensitive (left) and resistant (right) signature scores with curated HRD (homologous recombination deficiency) score across TCGA (The Cancer 
Genome Atlas) pan-solid tumor samples. PARPi sensitive and resistant signature scores were calculated by summarizing the Z-normalized log2RSEM (RNA-Seq by Expectation-Maximization) of the expression data for the genes in the PARPi sensitive and resistant signatures, based on the RNA-sequencing data of TCGA pan-solid tumor samples. THE curated HRD score was based on a previous study (Ref. 11). \#p < 0.01 (blue; negative correlation); ${ }^{*} \mathrm{p}<0.01$ (red; positive correlation).

2.3. The PARPi gene signatures facilitate the identification of cancer subtypes potentially suitable to PARPis

Next, we applied PARPis sensitivity and resistance gene signatures to both cancer cell lines and patient samples to identify cancer subtypes that are potentially vulnerable to PARPi.

Regarding the cancer cell lines, the results showed that cancer cells originating from the lineages of autonomic ganglia, bone, and lung display relatively high PARPi sensitive and low resistant score (Figure 3A; Suppl. Figure 1), suggesting that cancer cells with these lineages are more likely to be sensitive to PARPi treatment. Of note, there is high heterogeneity in the distribution of PARPi sensitivity and resistance scores across lung cancer cells (Figure 3A), implying that response to PARPis in lung cancer cells is most heterogeneous. Interestingly, a further investigation of the top 30 cell lines with high PARPi sensitive scores, we observed that small-cell lung cancer (SCLC) cell lines are highly enriched (Figure 3B), indicating that in lung lineage-derived tumors, SCLC other than non-SCLC (NSCLC), represent a potential subset vulnerable to PARPi. In support of this, SCLC has a significantly higher PARPi sensitive score but a lower resistant score than that of NSCLC (Figure 3C). Along the same lines, mining an independent data source from GDSC revealed that SCLC is more sensitive to the three clinically-approved PARPi Olaparib, Talazoparib, and Niraparib, compared with NSCLC (Figure 3D). Taken together, these results suggest that PARPi may represent a promising treatment for SCLC cells. Of important note, SCLC can be broadly divided into 4 molecular subtypes by differential expression of four key transcription regulators: achaete-scute homolog 1 (ASCL1), neurogenic differentiation factor 1 (NEUROD1), yes-associated protein 1 (YAP1), and POU class 2 homeobox 3 (POU2F3) [12]. We further found that only YAP1 expression is significantly correlated with the PARPi sensitive (negatively) and resistant (positively) signatures (Figure 3E), suggesting that the YAP1-driven SCLC subtype may be resistant to PARPis. In support of this, the YAP1-driven SCLC subtype is more resistant to PARPis, compared with other non-YAP1-driven SCLC subtypes, as indicated by a significantly higher AUC value of YAP1-driven SCLC cells in response to the three clinically-approved PARPi Olaparib and Talazoparib (Figure 3F). 
A

$$
\begin{aligned}
& \text { A }
\end{aligned}
$$

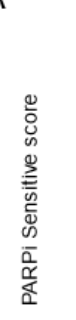
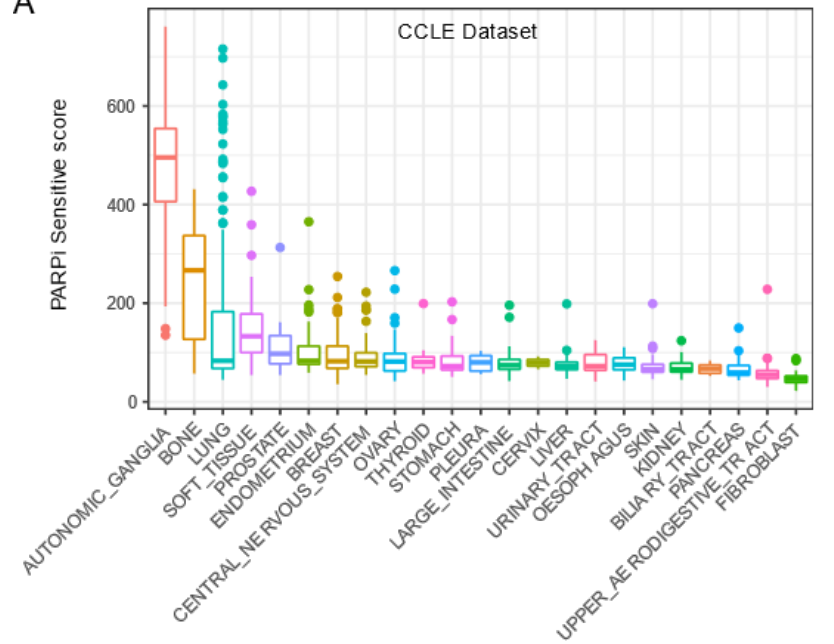

B

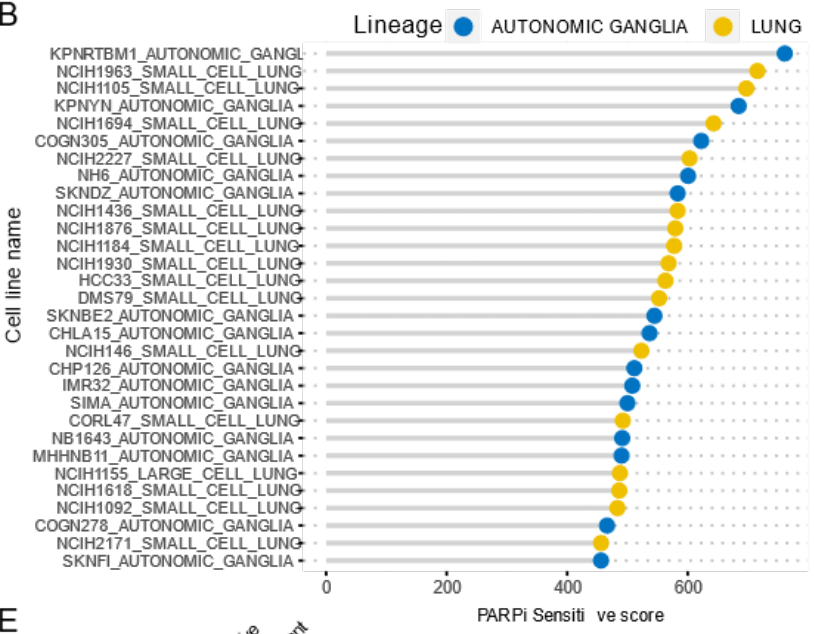

E

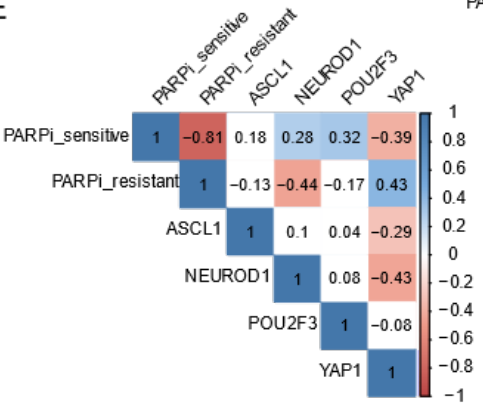

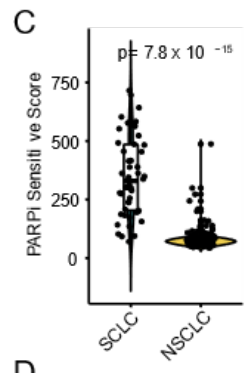
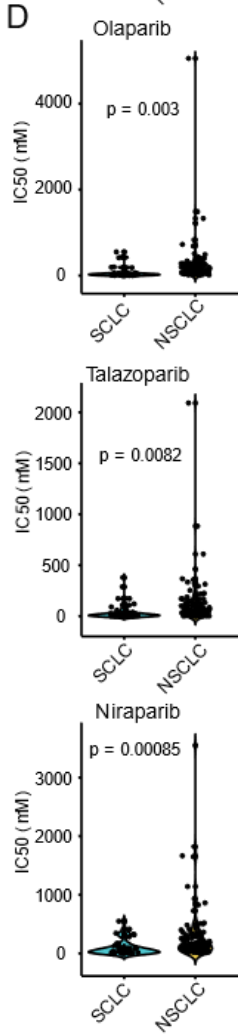

F

Figure 3. Identifying non-YAP1-driven SCLC as potential subsets for therapeutic response to PARP inhibitors (PARPis). A, Prospective analysis of PARPi sensitive signature score across CCLE (Cancer Cell Line Encyclopedia) pan-solid cancer lines. PARPi sensitive signature score was calculated by summarizing the Z-normalized log2RSEM (RNA-Seq by Expectation-Maximization) of the expression data for the genes in the PARPi sensitive and resistant signatures, based on the RNA-sequencing data of pan-solid cancer cell lines from CCLE; B, Top 30 cancer cell lines with high PARPi sensitive score. Note that cancer cells from SCLC (small-cell lung cancer) and autonomic ganglia are mainly enriched; C, Significant difference (by Welch's t-test) of PARPi sensitive signature score between SCLC and non-SCLC (NSCLC) cells from CCLE; D, Significant difference (by Welch's t-test) of IC50 [median inhibitory concentration (IC50); $\mu \mathrm{M}$ ] values between SCLC and non-SCLC (NSCLC) cells in response to three clinically-approved PARPis; E, Correlation matrix showing the correlation between PARPi signatures and four transcription factors that determine SCLC subtypes; Positive (in blue) and negative (in red) correlations are shown to the right, with color intensity and the size of the circle proportional to the correlation coefficient. Nonsignificant correlation coefficient values are left blank. On the right side of the correlogram, the legend color shows the correlation coefficients and the corresponding colors. p-value $<0.05$ is considered significant; $F$, The association of non-YAP1-driven SCLC with therapeutic responses to three clinically-approved PARPis. The characterization 
of SCLC cell lines was based on a previously curated data source (CellMiner-SCLC; https://discover.nci.nih.gov/SclcCellMinerCDB/), which was then merged with the corresponding drug profiles from GDSC. A significant difference was calculated by Welch's t-test.

Concerning the patient samples, we integrated the transcriptomic data from the pan-cancer cohort provided by The Cancer Genome Atlas (TCGA) project. Of note, for lung cancer samples, the TCGA project only contains NSCLC samples. We observed that tumors derived from the central nerves system, e.g., low-grade glioma (LGG) and glioblastoma (GBM), display the highest PARPi sensitive and lowest PARPi resistant signatures, whereas pancreatic tumor samples exhibit the highest PARPi resistant signature score (Figure 4A; Suppl. Figure 1B). In line with this, the cell-line-based models also revealed that cell lines derived from the pancreas are generally characterized by a high PARPi resistant signature score (Suppl. Figure 1A, B). Besides, clinical tumors (PCPG) from the autonomic ganglia lineage also exhibit a rather low PARPi resistant signature score, consistent with the cell-line-based models (Suppl. Figure 1A, B). 
A

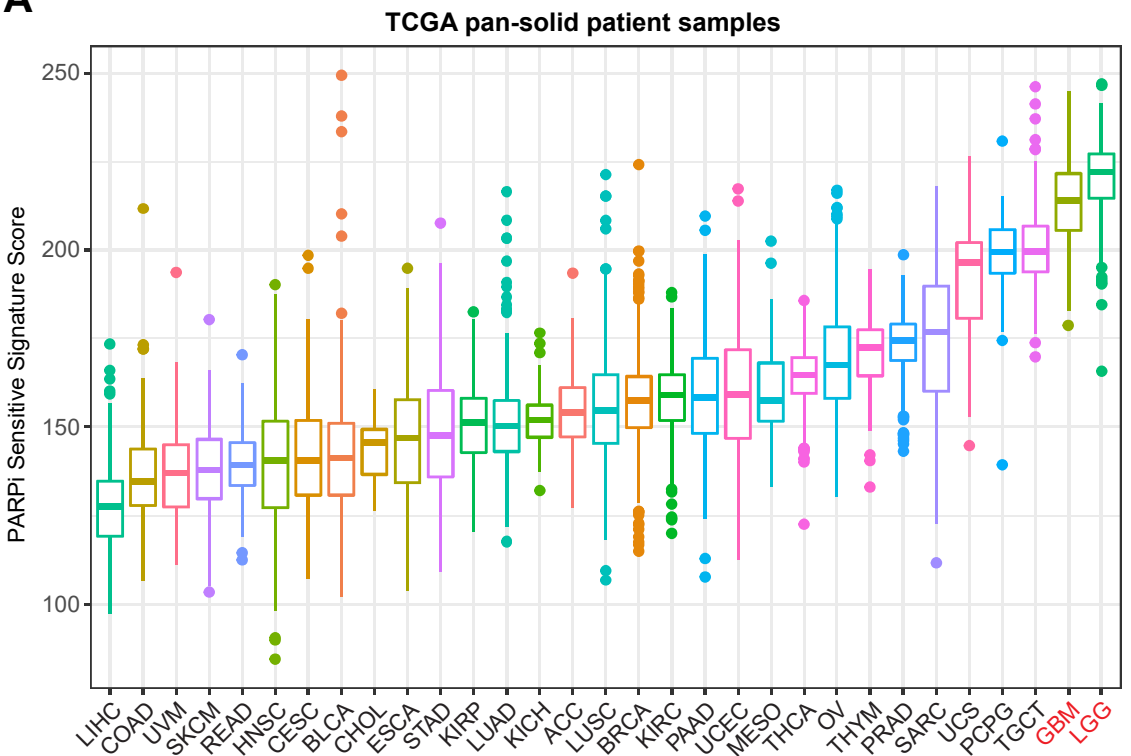

C
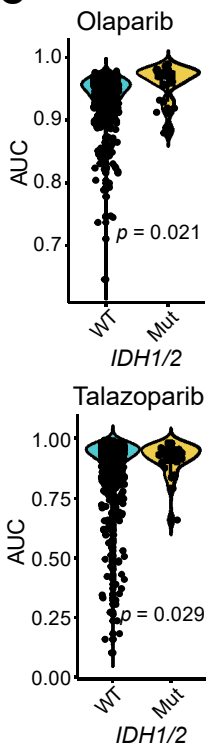

B
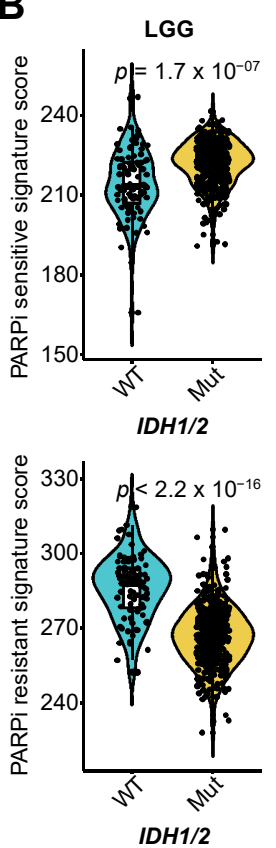

D
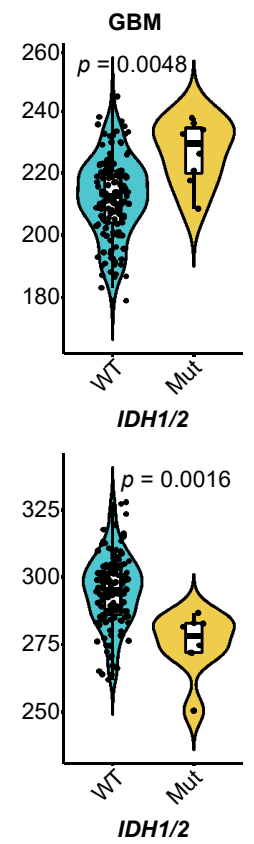

D LGG

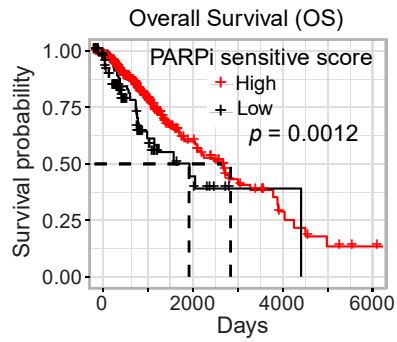

E

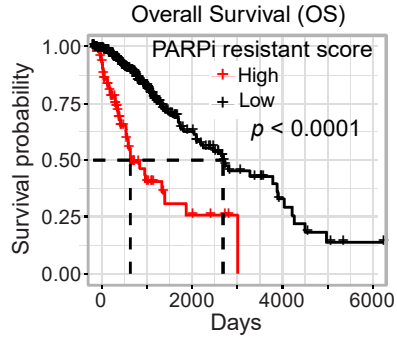

GBM

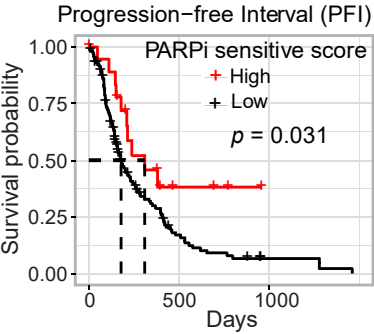

Progression-free Interval (PFI)

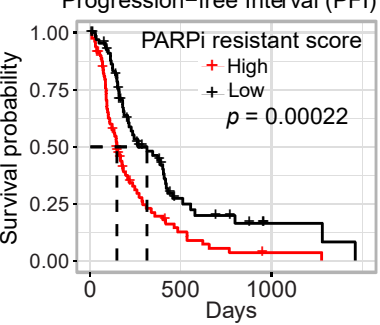

Figure 4. Identifying IDH1/2-mutant brain tumors as potential subsets for therapeutic response to PARPis. A, Prospective analysis of PARPi sensitive signature score across TCGA pan-solid cancer cohort. To note that brain tumors (LGG, low-grade glioma; GBM, glioblastoma multiforme) were ranked as the top cancer types with the highest PARPi sensitive signature score; B, Significant difference (by Welch's t-test) of PARPi sensitive and resistant signature scores between IDH1/2-mutant (mut) and wild-type (WT) samples of LGG and GBM; C, Significant difference (by Welch's t-test) in the sensitivity (reflected by AUC [area under the curve] of IDH1/2-mut and -WT solid cancer cells in response to two clinically-approved PARPis. D-E, Kaplan-Meier survival analyses of LGG (left) and GBM (right) stratified by the PARPi sensitive (upper panel) and resistant (lower panel) gene signatures.

It is well-known that mutations in isocitrate dehydrogenase 1 or 2 (IDH1/2), which results in the production of the oncometabolite 2-hydroxglutarate (2-HG), are the most common genetic alterations in glioma [13]. Interestingly, we observed that IDH1/2 mutations were significantly associated with high PARPi sensitive and low resistant scores in LGG and GBM tumor samples (Figure 4B), suggesting that IDH1/2-mutant tumors are likely to be more sensitive to PARPis. Supporting our findings, IDH1/2- 
mutant solid cancer cells have significantly lower AUC values compared with wild-type (WT) in response to PARPis Vorinostat and Belinostat, based on an independent GDSC data cohort (Figure 4C). Consistently, it was recently reported that IDH1/2-mutant tumors display higher sensitivity to the PARPis, compared with IDH-WT counterparts $[14,15]$. Furthermore, the PARPi resistant signature predicts a poor prognosis in terms of overall survival (OS) and progression-free survival (PFS) in patients with LGG and GBM (Figure 4D, E).

Taken together, non-YAP1-driven SCLC and IDH1/2-mutant brain tumors are potential cancer subsets suitable for PARP targeted therapy.

2.4. The PARPi resistant gene signature facilitates identifying drugs synergistically interacting with PARPi

The efficacy of PARPi treatment as a monotherapy is not durable, and intrinsic and acquired resistance are common in response to PARPi treatment. Thus, it is critical to identify additional targets to enhance the efficacy of PARPi treatment.

The genes in the established PARPi resistant signature represent potential effectors that mediate the intrinsic resistance to PARPi, implying that targeting these genes might sensitize cancer cells to PARP targeted therapy. Next, we sought to identify drug candidates whose effects correlated with the expression of these genes. Through individually correlating the 30 genes of the PARPi resistant signature (Figure 1C) with the drug compounds in the library, we observed that 10 of 30 genes whose higher expression significantly correlate with lower AUC values of drugs targeting EGFR-MAPK or SRC signaling pathways (Figure 5), e.g. EGFR inhibitors (Gefitinib, Erlotinib, Lapatinib, Afatinib, PD153035), MEK1/2 inhibitors (Trametinib, Selumetinib, PD318088), SRC inhibitors (Dasatinib, Saracatinib), suggesting cancer cells with high expression of these PARPi resistant genes are more sensitive to these targeted therapies. In support of this, previous evidence demonstrated that MEK inhibitors could artificially induce HRD and synergize with PARPi in multiple tumor types [16-18]. Further, recent evidence also revealed the PARP1 plays a critical role in mediating the acquired resistance to EGFR inhibitors, and PARPi could selectively eliminate the EGFR-resistant lung cancer cells [19]. Mechanistically, PARP-1 mediates the therapy resistance by regulating ROS levels independent of its classical role in DNA repair. More recently, pharmacological screening identified a synergistic interaction between Dasatinib and Olaparib in triple-negative breast cancer [20]. Collectively, these lines of evidence indicate the robustness of our established PARPi responsive (sensitive/resistant) signatures in identifying combined targets that synergistically augment the efficacy of PARPi. 

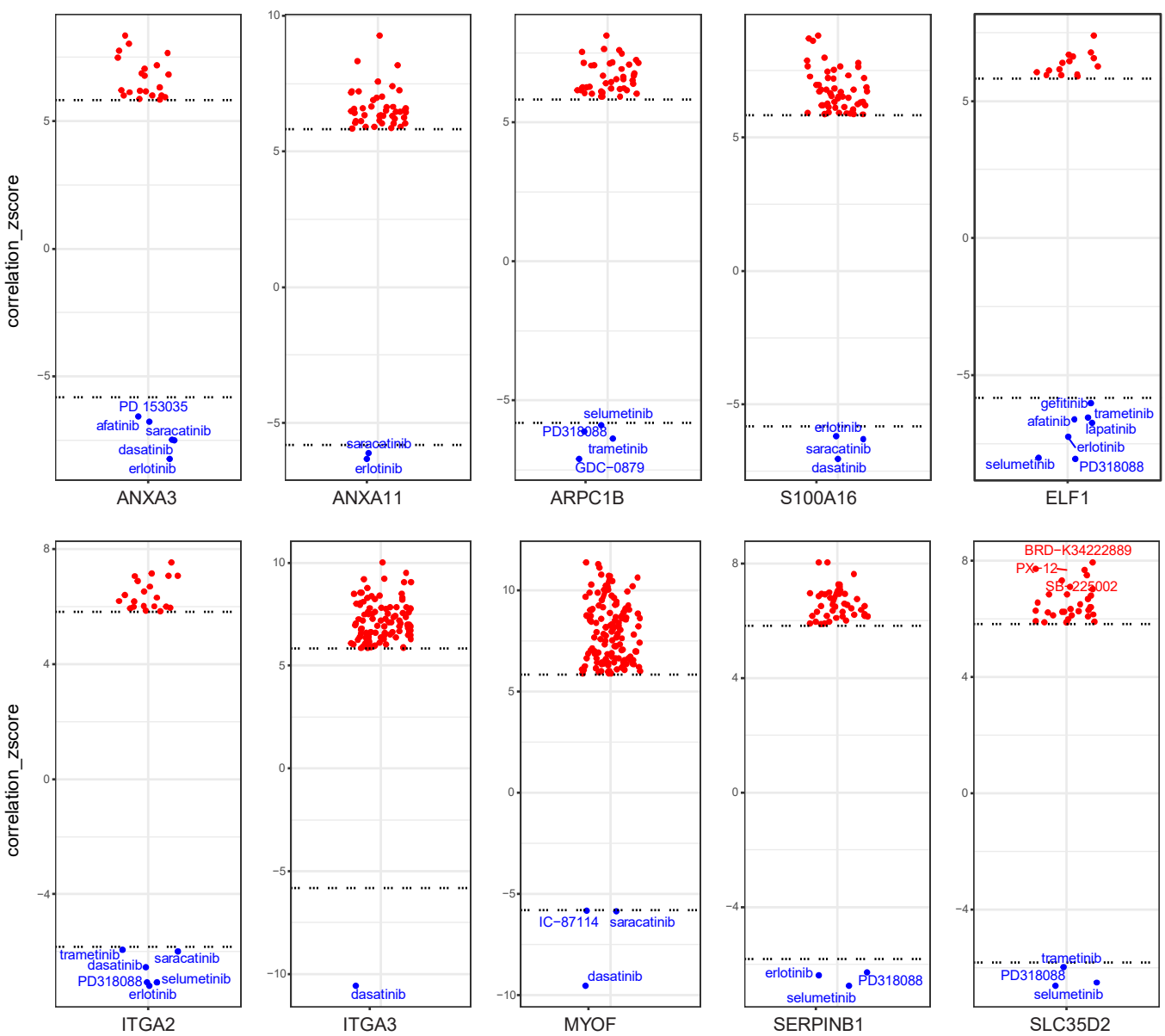

\begin{tabular}{|c|c|c|}
\hline EGFR inhibitors & MEK1/2 inhibitors & SRC inhibitors \\
\hline Gefitinib & Trametinib & Dasatinib \\
\hline Erlotinib & Selumetinib & Saracatinib \\
\hline Lapatinib & PD318088 & \\
\hline Afatinib & & \\
\hline PD153035 & & \\
\hline
\end{tabular}

Figure 5. Genes in the PARPi resistant signature correlate with the sensitivity of several clinically-approved inhibitors. Box-and-whisker plots show the extent of correlation between cytotoxic effects (reflected by the area under the curve [AUC] value) of drug compounds in the library $(n=481)$ and expression level of genes in the PARPi resistant signature. The y-axis indicates z scored Pearson's correlation coefficients; line, median; box, 25-75th percentile; whiskers, 2.5th and 97.5th percentile expansion; Here, significantly (adjusted $\mathrm{p}<0.05$ ) correlated inhibitors were shown (red dots: positively correlated; blue dots: negatively correlated). Note that cells with smaller AUC values are more sensitive to the tested drugs. Inhibitors targeting EGFR, MEK1/2, or SRC kinases are listed as the most negatively correlated drug.

\section{Discussion}

PARP represents a promising therapeutic target, given that PARPi exhibits synthetic lethality with tumors harboring HRD. Although a variety of HRD signatures have been developed to stratify cancer patients for PARP targeted therapies, the treatment response to PARPi is highly heterogeneous. Further, available HRD tests fail to consistently identify a subset of patients who can benefit from PARPis [21]. As such, better biomarkers that predict the therapeutic responses and facilitate the identification of subsets suitable for PARPis are critical. In this study, PARPi sensitive and resistance signatures were established by integrated pharmaco-transcriptomics of a clinically-approved PARPi Olaparib across a large cohort of solid cancer cell lines, which demonstrated that two potential patient subsets for PARPi monotherapy: IDH1/2-mutant brain tumors and non-YAP1-featured SCLC. We 
further identified several clinically-approved inhibitors as potential candidates that synergistically enhance the efficacy of PARPi in treating cancer.

Numerous gene signatures have been developed to predict the HRD to stratify patients for PARPi. However, in this study, our established PARPi signatures appear no correlation with curated HRD signature, which might be due to the fact that PARP has pleiotropic roles beyond DNA damage repair, such as ROS [19] or transcription regulation [22]. McGrail et al. [23] used gene expression profiles from ovarian and breast cancer cell lines that display high sensitivity to olaparib and rucaparib, leading to deriving a transcriptional algorithm that can improve the prediction sensitivity to PARP inhibitors. In line with this, recent studies support the expansion of PARP-targeted therapy in cancer irrespective of classical HRD signatures [5-7]. In a recent phase III clinical trial recruiting patients with newly diagnosed advanced ovarian cancer who had a response to platinum-based chemotherapy, the therapeutic benefits from PARPi niraparib is regardless of the presence or absence of HRD [5].

Interestingly, based on the HRD-independent PARPi signature, we identified IDH1/2-mutant brain tumors and SCLC as the potential subsets for PARPi. Our findings were in line with recent studies demonstrating that IDH1/2-mutant tumors display higher sensitivity to the PARPis, compared with IDH-WT counterparts [14,15]. Mechanistically, IDH1/2 mutations induce an HRD phenotype via inhibition of specific $\alpha \mathrm{KG}$-dependent dioxygenases, consequently rendering IDH1/2-mutant tumors vulnerable to PARPi [15]. In SCLC, previous evidence showed that most SCLC cell lines tested were highly sensitive to PARPi compared with NSCLC cell lines. PARPi has also been widely investigated in SCLC [24]. However, PARPi drug sensitivity was not universal for all SCLC cell lines tested. Interestingly, our study reveals that the YAP1-driven SCLC subtype predicts resistance to PARPi, which might explain the heterogeneous responses observed. Thus, more molecularly based stratifications for PARP targeted therapy in SCLC patients are needed [25].

Intrinsic and acquired resistance to PARPi is common, leading to extensive pursuits for combination therapy to improve the efficacy of PARPi [26]. In this study, based on the limited drug library $(n=481)$, we identified that cancer cells with high expression of PARPi resistant genes are more sensitive to inhibitors targeting EGFR, MEK1/2, or SRC kinases, which was in line with previous evidence demonstrating that PARP1 mediates the acquired resistance to EGFR inhibitors in NSCLC through controlling ROS levels [19]. This study highlighted DNA repair-independent role of PARPi. Likewise, MEK inhibitors could also synergize with PARPi in multiple tumor types [16-18], largely due to its artificial induction of HRD. Further, the synergism between Dasatinib and Olaparib was validated in a previous study [20]. These lines of evidence suggest the robustness of our established PARPi signature. Due to the limited size of the current drug library in this study, further analyses based on a large cohort of drug compounds, particularly clinically-approved ones, are required.

\section{Materials and Methods}

\subsection{Public Databases}

Processed drug screening and gene expression data across a set of small-molecule compounds $(n=481)$ and solid cancer cell lines $(n=659)$ from a published study $[27,28]$ were downloaded for reanalysis. Correlation data across all 481 small molecules against individual transcriptomes that are significantly correlated with response to at least one small molecule were included for analysis $[27,28]$. The area under the curve (AUC), determined by fitted concentration-response curves (2-fold dilution, over a 16-point concentration range), is used as a measure of sensitivity. Fisher's z-transformation was applied to the correlation coefficients to adjust for (normalize) variations in cancer cell line number across small molecules and contexts [27,28]. For validation analysis, the drug response profiles to different PARPis from independent GDSC (Genomics of Drug Sensitivity in Cancer, https://www.cancerrxgene.org/) dataset were employed. The normalized RNA-sequencing data of identified genes across The Cancer Genome Atlas (TCGA) Pan-cancer cohort was downloaded from cBioPortal (https://www.cbioportal.org/). Normalized transcriptomic data of cancer cell lines were 
downloaded from Cancer Cell Line Encyclopedia (CCLE) project (https://portals.broadinstitute.org/ccle). Characterization of SCLC subtypes was based on a previously curated public resource (CellMiner-SCLC; https://discover.nci.nih.gov/SclcCellMinerCDB/) [29].

\subsection{PARPi and HRD gene signature scores}

PARPi sensitive and resistant signature scores were calculated by summarizing the Z-normalized $\log$ 2RSEM (RNA-Seq by Expectation-Maximization) of the expression data for the genes in the PARPis sensitive and resistant signatures, based on the RNA-sequencing data of TCGA pan-solid patients' tumor and pan-solid cancer cell lines from CCLE [30,31].

The curated HRD gene signature score was based on a TCGA pan-cancer project [11].

\subsection{Survival Analysis}

Survival analysis was performed using "survminer" and "survival" $\mathrm{R}$ packages. Tumor samples within the TCGA Pan-cancer, LGG, and MESO cohorts were divided into two groups, based on the best-separation cut-off value of indicated genes to plot the Kaplan-Meier survival curves [30,32].

All analyses were performed using $\mathrm{R}$ (version 3.6.3).

\section{Conclusions}

The established PARPi responsive (sensitive/resistant) signatures in solid tumors exhibit robustness in identifying cancer subtypes that are potentially vulnerable to PARP-targeted therapy, and combined targets that synergistically augment the efficacy of PARPi.

Supplementary Materials: Figure S1: Prospective analysis of PARPi resistant signature score across CCLE (Cancer Cell Line Encyclopedia) pan-solid cancer lines and TCGA (The Cancer Genome Atlas) pan-solid tumor samples.

Author Contributions: Conceptualization, Haitang Yang and Feng Yao. Methodology, Haitang Yang and Beibei Sun. Formal Analysis, Haitang Yang and Beibei Sun. Investigation, Haitang Yang, Beibei Sun, Ke Xu, Tuo Zhang. Data Curation, Sean R.R. Hall, Ke Xu, Liang Zhao, Swee Tan, Ralph Schmid, Ren-Wang Peng. Writing - Original Draft Preparation, Haitang Yang. Writing - Review \& Editing, all authors. Supervision, Haitang Yang and Feng Yao. Project Administration, Feng Yao. Funding Acquisition, Feng Yao. All authors have read and agreed to the published version of the manuscript.

Funding: This research received no external funding.

Acknowledgments: This study used TCGA Program database. The interpretation and reporting of these data are the sole responsibility of the authors. The authors acknowledge the efforts of the National Cancer Institute.

Conflicts of Interest: The authors declare no conflict of interest.

\section{References}

1. Hanahan, D.; Weinberg, R.A. Hallmarks of cancer: the next generation. Cell 2011, 144, 646-674, doi:10.1016/j.cell.2011.02.013.

2. Fong, P.C.; Boss, D.S.; Yap, T.A.; Tutt, A.; Wu, P.; Mergui-Roelvink, M.; Mortimer, P.; Swaisland, H.; Lau, A.; O'Connor, M.J., et al. Inhibition of poly(ADP-ribose) polymerase in tumors from BRCA mutation carriers. N Engl J Med 2009, 361, 123-134, doi:10.1056/NEJMoa0900212.

3. Luo, X.; Kraus, W.L. On PAR with PARP: cellular stress signaling through poly(ADP-ribose) and PARP1. Genes Dev 2012, 26, 417-432, doi:10.1101/gad.183509.111.

4. Bai, P. Biology of Poly(ADP-Ribose) Polymerases: The Factotums of Cell Maintenance. Mol Cell 2015, 58, 947-958, doi:10.1016/j.molcel.2015.01.034.

5. Gonzalez-Martin, A.; Pothuri, B.; Vergote, I.; DePont Christensen, R.; Graybill, W.; Mirza, M.R.; McCormick, C.; Lorusso, D.; Hoskins, P.; Freyer, G., et al. Niraparib in Patients with Newly Diagnosed Advanced Ovarian Cancer. N Engl J Med 2019, 381, 2391-2402, doi:10.1056/NEJMoa1910962.

6. Smeby, J.; Kryeziu, K.; Berg, K.C.G.; Eilertsen, I.A.; Eide, P.W.; Johannessen, B.; Guren, M.G.; Nesbakken, A.; Bruun, J.; Lothe, R.A., et al. Molecular correlates of sensitivity to PARP inhibition beyond homologous 
recombination deficiency in pre-clinical models of colorectal cancer point to wild-type TP53 activity. EBioMedicine 2020, 59, 102923, doi:10.1016/j.ebiom.2020.102923.

7. Pilie, P.G.; Gay, C.M.; Byers, L.A.; O'Connor, M.J.; Yap, T.A. PARP Inhibitors: Extending Benefit Beyond BRCA-Mutant Cancers. Clin Cancer Res 2019, 25, 3759-3771, doi:10.1158/1078-0432.CCR-18-0968.

8. Lord, C.J.; Ashworth, A. PARP inhibitors: Synthetic lethality in the clinic. Science 2017, 355, 1152-1158, doi:10.1126/science.aam7344.

9. Noordermeer, S.M.; van Attikum, H. PARP Inhibitor Resistance: A Tug-of-War in BRCA-Mutated Cells. Trends Cell Biol 2019, 29, 820-834, doi:10.1016/j.tcb.2019.07.008.

10. Li, H.; Liu, Z.Y.; Wu, N.; Chen, Y.C.; Cheng, Q.; Wang, J. PARP inhibitor resistance: the underlying mechanisms and clinical implications. Mol Cancer 2020, 19, 107, doi:10.1186/s12943-020-01227-0.

11. Knijnenburg, T.A.; Wang, L.; Zimmermann, M.T.; Chambwe, N.; Gao, G.F.; Cherniack, A.D.; Fan, H.; Shen, H.; Way, G.P.; Greene, C.S., et al. Genomic and Molecular Landscape of DNA Damage Repair Deficiency across The Cancer Genome Atlas. Cell Rep 2018, 23, 239-254 e236, doi:10.1016/j.celrep.2018.03.076.

12. Rudin, C.M.; Poirier, J.T.; Byers, L.A.; Dive, C.; Dowlati, A.; George, J.; Heymach, J.V.; Johnson, J.E.; Lehman, J.M.; MacPherson, D., et al. Molecular subtypes of small cell lung cancer: a synthesis of human and mouse model data. Nat Rev Cancer 2019, 19, 289-297, doi:10.1038/s41568-019-0133-9.

13. Zhang, C.; Moore, L.M.; Li, X.; Yung, W.K.; Zhang, W. IDH1/2 mutations target a key hallmark of cancer by deregulating cellular metabolism in glioma. Neuro Oncol 2013, 15, 1114-1126, doi:10.1093/neuonc/not087.

14. Molenaar, R.J.; Radivoyevitch, T.; Nagata, Y.; Khurshed, M.; Przychodzen, B.; Makishima, H.; Xu, M.; Bleeker, F.E.; Wilmink, J.W.; Carraway, H.E., et al. IDH1/2 Mutations Sensitize Acute Myeloid Leukemia to PARP Inhibition and This Is Reversed by IDH1/2-Mutant Inhibitors. Clin Cancer Res 2018, 24, 1705-1715, doi:10.1158/1078-0432.CCR-17-2796.

15. Sulkowski, P.L.; Corso, C.D.; Robinson, N.D.; Scanlon, S.E.; Purshouse, K.R.; Bai, H.; Liu, Y.; Sundaram, R.K.; Hegan, D.C.; Fons, N.R., et al. 2-Hydroxyglutarate produced by neomorphic IDH mutations suppresses homologous recombination and induces PARP inhibitor sensitivity. Sci Transl Med 2017, 9, doi:10.1126/scitranslmed.aal2463.

16. Vena, F.; Jia, R.; Esfandiari, A.; Garcia-Gomez, J.J.; Rodriguez-Justo, M.; Ma, J.; Syed, S.; Crowley, L.; Elenbaas, B.; Goodstal, S., et al. MEK inhibition leads to BRCA2 downregulation and sensitization to DNA damaging agents in pancreas and ovarian cancer models. Oncotarget 2018, 9, 11592-11603, doi:10.18632/oncotarget.24294.

17. Maertens, O.; Kuzmickas, R.; Manchester, H.E.; Emerson, C.E.; Gavin, A.G.; Guild, C.J.; Wong, T.C.; De Raedt, T.; Bowman-Colin, C.; Hatchi, E., et al. MAPK Pathway Suppression Unmasks Latent DNA Repair Defects and Confers a Chemical Synthetic Vulnerability in BRAF-, NRAS-, and NF1-Mutant Melanomas. Cancer Discov 2019, 9, 526-545, doi:10.1158/2159-8290.CD-18-0879.

18. Sun, C.; Fang, Y.; Yin, J.; Chen, J.; Ju, Z.; Zhang, D.; Chen, X.; Vellano, C.P.; Jeong, K.J.; Ng, P.K., et al. Rational combination therapy with PARP and MEK inhibitors capitalizes on therapeutic liabilities in RAS mutant cancers. Sci Transl Med 2017, 9, doi:10.1126/scitranslmed.aal5148.

19. Marcar, L.; Bardhan, K.; Gheorghiu, L.; Dinkelborg, P.; Pfaffle, H.; Liu, Q.; Wang, M.; Piotrowska, Z.; Sequist, L.V.; Borgmann, K., et al. Acquired Resistance of EGFR-Mutated Lung Cancer to Tyrosine Kinase Inhibitor Treatment Promotes PARP Inhibitor Sensitivity. Cell Rep 2019, 27, $3422-3432$ e3424, doi:10.1016/j.celrep.2019.05.058.

20. Corrales-Sanchez, V.; Noblejas-Lopez, M.D.M.; Nieto-Jimenez, C.; Perez-Pena, J.; Montero, J.C.; Burgos, M.; Galan-Moya, E.M.; Pandiella, A.; Ocana, A. Pharmacological screening and transcriptomic functional analyses identify a synergistic interaction between dasatinib and olaparib in triple-negative breast cancer. J Cell Mol Med 2020, 24, 3117-3127, doi:10.1111/jcmm.14980.

21. Miller, R.E.; Leary, A.; Scott, C.L.; Serra, V.; Lord, C.J.; Bowtell, D.; Chang, D.K.; Garsed, D.W.; Jonkers, J.; Ledermann, J.A., et al. ESMO recommendations on predictive biomarker testing for homologous recombination deficiency and PARP inhibitor benefit in ovarian cancer. Ann Oncol 2020, 31, 1606-1622, doi:10.1016/j.annonc.2020.08.2102.

22. Kraus, W.L.; Hottiger, M.O. PARP-1 and gene regulation: progress and puzzles. Mol Aspects Med 2013, 34 , 1109-1123, doi:10.1016/j.mam.2013.01.005.

23. McGrail, D.J.; Lin, C.C.; Garnett, J.; Liu, Q.; Mo, W.; Dai, H.; Lu, Y.; Yu, Q.; Ju, Z.; Yin, J., et al. Improved prediction of PARP inhibitor response and identification of synergizing agents through use of a novel gene expression signature generation algorithm. NPJ Syst Biol Appl 2017, 3, 8, doi:10.1038/s41540-0170011-6. 
24. Byers, L.A.; Wang, J.; Nilsson, M.B.; Fujimoto, J.; Saintigny, P.; Yordy, J.; Giri, U.; Peyton, M.; Fan, Y.H.; Diao, L., et al. Proteomic profiling identifies dysregulated pathways in small cell lung cancer and novel therapeutic targets including PARP1. Cancer Discov 2012, 2, 798-811, doi:10.1158/2159-8290.CD-12-0112.

25. Farago, A.F.; Yeap, B.Y.; Stanzione, M.; Hung, Y.P.; Heist, R.S.; Marcoux, J.P.; Zhong, J.; Rangachari, D.; Barbie, D.A.; Phat, S., et al. Combination Olaparib and Temozolomide in Relapsed Small-Cell Lung Cancer. Cancer Discov 2019, 9, 1372-1387, doi:10.1158/2159-8290.CD-19-0582.

26. Barayan, R.; Ran, X.; Lok, B.H. PARP inhibitors for small cell lung cancer and their potential for integration into current treatment approaches. J Thorac Dis 2020, 12, 6240-6252, doi:10.21037/jtd.2020.03.89.

27. Rees, M.G.; Seashore-Ludlow, B.; Cheah, J.H.; Adams, D.J.; Price, E.V.; Gill, S.; Javaid, S.; Coletti, M.E.; Jones, V.L.; Bodycombe, N.E., et al. Correlating chemical sensitivity and basal gene expression reveals mechanism of action. Nat Chem Biol 2016, 12, 109-116, doi:10.1038/nchembio.1986.

28. Yang, H.; Zhao, L.; Gao, Y.; Yao, F.; Marti, T.M.; Schmid, R.A.; Peng, R.W. Pharmacotranscriptomic Analysis Reveals Novel Drugs and Gene Networks Regulating Ferroptosis in Cancer. Cancers (Basel) 2020, 12, doi:10.3390/cancers12113273.

29. Tlemsani, C.; Pongor, L.; Elloumi, F.; Girard, L.; Huffman, K.E.; Roper, N.; Varma, S.; Luna, A.; Rajapakse, V.N.; Sebastian, R., et al. SCLC-CellMiner: A Resource for Small Cell Lung Cancer Cell Line Genomics and Pharmacology Based on Genomic Signatures. Cell Rep 2020, 33, 108296, doi:10.1016/j.celrep.2020.108296.

30. Yang, H.; Xu, D.; Yang, Z.; Yao, F.; Zhao, H.; Schmid, R.A.; Peng, R.W. Systematic Analysis of Aberrant Biochemical Networks and Potential Drug Vulnerabilities Induced by Tumor Suppressor Loss in Malignant Pleural Mesothelioma. Cancers (Basel) 2020, 12, doi:10.3390/cancers12082310.

31. Yang, H.; Yao, F.; Marti, T.M.; Schmid, R.A.; Peng, R.W. Beyond DNA Repair: DNA-PKcs in Tumor Metastasis, Metabolism and Immunity. Cancers (Basel) 2020, 12, doi:10.3390/cancers12113389.

32. Yang, H.; Liang, S.Q.; Xu, D.; Yang, Z.; Marti, T.M.; Gao, Y.; Kocher, G.J.; Zhao, H.; Schmid, R.A.; Peng, R.W. HSP90/AXL/eIF4E-regulated unfolded protein response as an acquired vulnerability in drugresistant KRAS-mutant lung cancer. Oncogenesis 2019, 8, 45, doi:10.1038/s41389-019-0158-7.

(C) 2020 by the authors. Submitted for possible open access publication under the terms and conditions of the Creative Commons Attribution (CC BY) license (http://creativecommons.org/licenses/by/4.0/). 
A

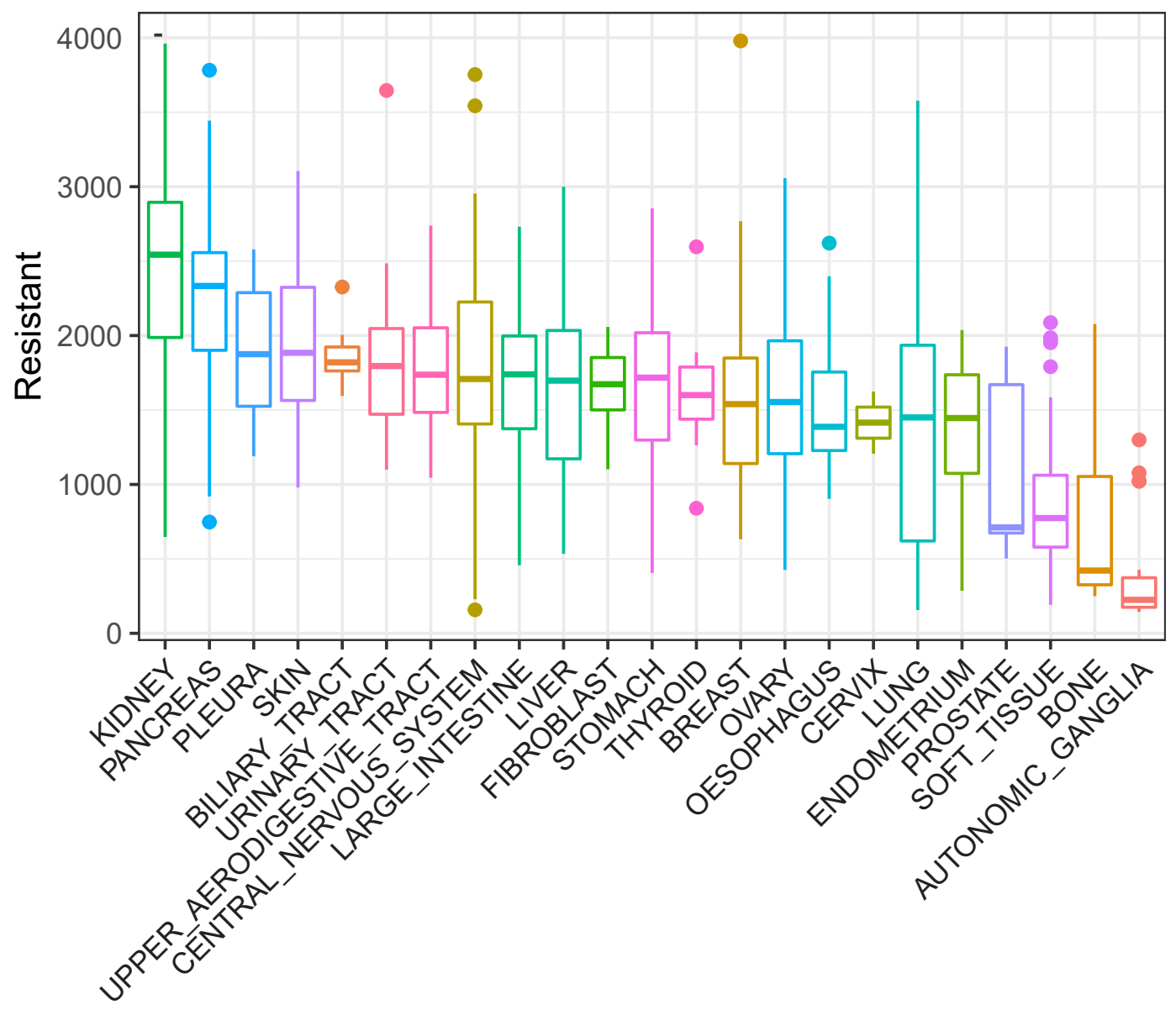

B

TCGA pan-solid patient samples

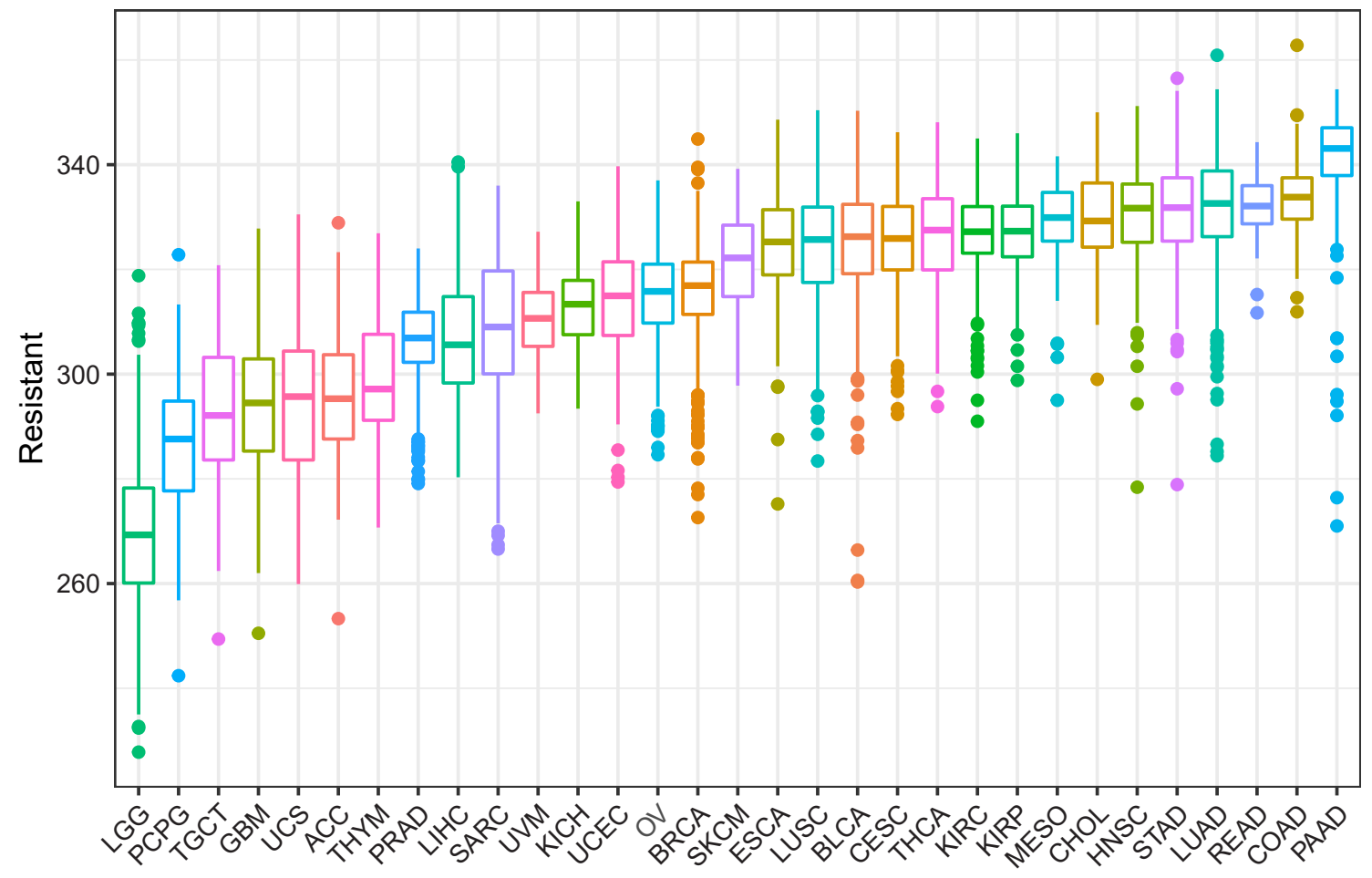

